werden, glaubt $\mathrm{Th}$. We y ${ }^{\text {(5) }}$ berechtigt zu sein, den Schluß zu ziehen, daß die Fällbarkeit der Proteine durch Azeton am besten sich erklären lagt durch die Fällbarkeit der Aminosăuren.

Dem Gedankengange von Th. We yl folgend, mubte man annehmen, dal die Fällbarkeit der Proteine durch Alkohol denselben Grund haben würde, wie für Azeton. Und in der Tat sind auch die Amidosäuren: Glykokoll, Alaun, Asparaginsäure, Glutaminsäure, infolge ihrer geringen Löslichkeit in Alkohol durch letzteren fällbar. Ebenso kann man voraussetzen, daA auch die Peptide, soweit man sie aus den Untersuchungen E. Fis cher's kennt, ebenfalls im Akohol schwer loslich sind und daher auch aus ihren wässerigen Lobsungen durch Alkohol ausgefalit werden können. Jedoch fehlen die direkten Versuche. Aus eigener Erfahrung habe ich mich überzeugt, daB das Tripeptid, Leuzyl - Glyzyl -- Glyzin, aus drciprozentiger wässeriger Losung durch Alkohol selbst bei beträchtlichem Ueberschusse $n$ ich t gefällt wird; bei einem Zusatz von etwa $1 / 40 \mathrm{Vol}$. Aether entstand sofortige Ausfällung.

St. Fetersburg, K. Inst.f. experim. Med. 1910.

3) Th. We y 1, Zeitschr. t. physiol. Chem. 65, 248.

\title{
Ueber die Wirkung von Radiumstrahlen auf Kolloide.
}

\author{
Von W. P. Jorissen und H. W. Woudstra. (Fingegangen am 1. Nov. 1910)
}

1. W. B.Hard $y^{1}$ ) war der erste, der die Einwirkung von Radium auf eine kolloide Lösung untersucht hat. In den "Proc, of the Cambr. Phil. Soc." berichtet er unter dem Titel "On the influence of electrons on colloidal solutions": "Specially purified globulin from blood was dissolved (a) in a trace of acetic acid, (b) in a trace of sodium hydrate. In presence of acetic acid the globulin was found to move in an electric field from anode to cathode, in presence of alkali it moved from cathode to anode. In the former case therefore the globulin particles carried a positive charge, in the latter a negative charge. These two solutions were exposed to the radiations from radiumbromide and it was found that the electronegative solution of globulin was turned into an opaque jelly in $\mathbf{3}$ minutes, while the electropositive solution became inore mobile and less opalescent."

Mehr teilte er nicht mit. Wahrscheinlich ist das Radiumpräparat unbedeckt benutzt worden, denn E. Rutherford ${ }^{2}$ ) sagt, nachdem er W.B.Hardy's Versuche erwähnt hat: "These actions were found to be due to the ays of radium alone. This is further evidence in favour of the view that the $\alpha$ rays consist of projected positively charged bodies of atomic dimensions, for a similar coagulation effect is produced by the

1) Proc Cambr. Phil. Suc 12, 201 May 18, 1903). Proc. Physiol. Soc (May 16, 1903). l.etztgenannte Mitteilung huben wir nicht in Handeu gehabt. Sie wird, weil sie zwei Tagre vor der andern gemacht wurde, wathl dieselbe scin als die erstgenannte.

3) Radio-Activity, second edition (Cambridge 1905). 215 metallic ions of liquid electrolytes, and has been shown by W. C. D. Weth a m ${ }^{3}$ ) to be due to the electric charge carried by the ions."

Ein Jahr später als W. B. Hardy teilten Victor Henri und André Mayer") die Resultate ihrer Versuche mit. Sie beschreiben diese wie folgt:

.Actions sur les colloïdes simples. Les radiations $\boldsymbol{f}$ (chargées négativement) peuvent précipiter les colloïdes positifs et sont sans action sur les colloïdes négatifs. Nous avons expérimenté sur l'argent colloïdal (négatif) et l'hydrate ferrique (positif): $2 \mathrm{ccm}$ du colloilde étaient soumis à l'action des radiations du sel de radium contenue dans un tube de verre bouché. et conservés dans une boîte de plomb à temperature constante $(25 \%)$. Après 4 jours dexposition, les colloïdes sont intacts. Mais si, a chacun d'eux, on ajoute une quantité d'électrolyte (azotate de soude) insuffisante pour amener la précipitation et yu'on expose les colloüdes ainsi sensibilisés à l'action des radiations, le colloïde negatif demeure intact, le colloïde positif est précipité."

Fs ist schade, dab Victor Henri und André Mayer nicht erwähnen. ob sie zu gleicher Zeit Kontrollversuche angestelit haben mit denselben Solen unter gleichen Umständen (also mit derselben Quantität Natritumnitrat. aber

3) Phil Mag. (Nov. 1899); Theory of Solutions (Cambridge 1902), 326.

4) Compt. rend. 138, 521 (1904). Für die von ihnen ebenfalls unterstchteWirkung aui $H$ ämoglobin, Fermente und rote Blutkorperchen sei hingewiesen auf die zitlerte Ablandiung. 
ohne Radium). Sie benutzten ungefăhr $100 \mathrm{mg}$ Radiumbromid, zu ihrer Verfügung gestellt von P. Curie; über die Aktivität des Pråparats wird nichts mitgeteilt.

In selben $\mathrm{Jahr}^{5}$ ) wiederholten sie ihre Versuche, wobei $0,08 \mathrm{~g}$ reines Radiumbromid von P. Curie gebraucht wurde. Sie untersuchten drei positive Kolloide (Eisenhydroxyd, Magdalarot und Methylviolett) und drei negative (kolloides Silber, Kupferferrozyanid und Anilinblau); $2 \mathrm{ccm}$ der Sole wurden in einem Glasröhrchen der Wirkung der $\boldsymbol{\beta}$-Strahlen des Radiums ausgesetzt. - Nach fünf Tagen waren die positiven Kolloide präzipitiert, die negativen nicht. Weitere Einzelheiten und Kontrollversuche werden nicht angegeben.

Aus der folgenden Mitteilung von G. Dreyer und $\mathrm{O} . \mathrm{Hanssen}{ }^{6}$ ) kann man keinen SchluB ziehen. weil sie nicht mitteilen, auf welche Weise sie das Radium anwendeten und auch nicht, ob ihre Sole positiv oder negativ waren. Sie sagen nur: "L'albumine le plus coagulable à la lumière, le vitelline, se coagule aussi à l'éclairement de radium. Nous n'avons trouvé rien de semblable pour la globuline, le fibrinogène, ni pour des solutions de ricine, de trypsine, de présure, de coliagglutine, quoique pour toutes ces substances le pouvoir d'agglutiner le sang et les bactéries et l'action enzymatique s'affaiblissent aussi - plus ou moins suivant les cas, c'est vrai - a l'éclairement de radium."

Aus dem von A. Righ ${ }^{\top}{ }^{T}$ in seiner Rektoratsrede Mitgeteilten über die Wirkung von Radium auf Kolloide kann man nicht schlleben, ob es sich auf die Versuche von W.B. Hardy und von Victor Henri und André Mayer bezieht, oder aber auf eigene Versuche. Er sagt - wir benutzen hier N. Pa p padà's ") Uebersetzung -: "Wenn man eine Lösung eines negativen Kolloids (z. B. des Alkali-Globulins des Ochsenserums) der Strahlungswirkung eines Radiumsalzes aussetzt, so erhält man eine allmähliche (graduelle) Koagulation, während dies nicht geschieht, wenn man statt dessen in dem Versuch ein positives Kolloid anwendet, welches vielmehr manchmal noch flüssiger zu werden scheint. Da die $\boldsymbol{\beta}$-Strahlen, welche die Flüssigkeit durchdringen, nur zum kleinsten Teil ab-

3) Compt. rend. de la Suc, de Biol, 57, 33 (1904). A rthur Müller (Allgem. Chemie der Kolloide [1907). 82) spricht, als et diese Versuche erwatint, von der Wirkung negativ geladener X-Strahlen (?)

9 Compt. tend. 145, 234 (1907).

$\eta$ Lt nuove vedute sull' intima struttura della materia (Bologna 1908)

b) Koll.-Zeitschr. 4. 214 (1909). sorbiert werden, die $\propto$-Strahlen aher vollständig, so ist es natürlich, die Koagulation den elektrischen Ladungen zuzuschreiben, die das Kolloid von diesen letzteren empfangen hat. LäBt man dagegen auf ein positives Kolloid (z. B. Eisenhydroxyd) nicht mehr die $\alpha$-Strahlen, sondern nur die $\beta$-Strahlen einwirken, so erhält man ebenfalls, wenn auch langsamer, Koagulation.* C. Doelter\%, der die Verfügung hatte über „ein Fläschchen mit 0,5 g Radiumchlorid“ aus der bekannten Sammlung der Kaiserl. Akademis inWien, teilt seineVersuche und die bekommenen Resultate sehr kurzgefaBt mit ${ }^{10}$ ).

Er untersuchte Sole von Mangan-, Aluminium-. Chrom- und Eisenhydroxyd, Natriumsilikat, Arsenund Antimontrisulfid und Selen. Die Sole wurden gewöhnlich während 35 Tagen der Wirkung des Radiums ausgesetzt; bei Aluminium- und Chromhydroxyd und bei Selen, wurden die Versuche mehrmals wiederholt und sie dauerten dann 6 bis 100 Tage.

Während aus dem nichtbestrahiten Selensol nichtkristallisiertes Selen sich absetzte, kam aus dem bestrahlten Sol nach 100 Tagen kristallisiertes Selen zum Vorschein.

Die Mangan-, Chrom-, Aluminium- und Eisenhydroxydsole wurden nicht koaguliert, selbst nicht nachdem sie während 100 Tagen bestrahlt worden waren. Bei Aluminumhydroxyd war das Resultat nach 6 Tagen, während ,eine kleine Menge eines Elektrolyten, nämlich Salmiak. unter dem Schwellenwert " zugefügt worden war. ebenfalls negativ.

Das Arsentrisulfid und das Antimontrisulfid gaben nach 100 Tagen einen geringen Niederschlag. Das Natriumsilikatsol koagulierte.

Ein Chlornatriumsol (in Benzol) war nach 10 Tagen teilweise koaguliert. Ein Hydrosol von Goldpurpur wurde nicht niedergeschlagen, ebensowenig eine kolloide Lösung von Ilsemannit $\left(\mathrm{MoO}_{2}+4 \mathrm{MoO}_{3}\right)$. Organische Parbstoffe zeigten keine Koagulation “ - fügt er hinzu -. "auch bei Wein trat sie nicht ein, ebensowenig war Blut koaguliert, doch waren die roten Blutkörperchen kompakter."

Aus C. Doelter's Beobachtungen kann man. wenigstens soweit er diese mitteilte, nicht vieles schlieben. Bei demVersuch mit Aluminium oxydsol und Salmiak wird nicht mitgeteilt, ob man in der Nähe des "Schwellenwertes" war, oder nicht. "Die nicht belichtete Kontrollprobe" - fügt er nur hinzu -- nergab keine Ver änderung. "

9) Vas Kadium und die Farben (Dresden 1911) 
Dab die Arsen- und Antimontrisulfidsole nach 100 Tagen "einen sehr geringen Niederschlag" bezw. "einen gelartigen Niederschlag in geringer Menge" zeigten, kann nicht befremden, wenn man die ziemlich kleine Stabilität dieser Sole kennt. Kontrollversuche werden überdies nicht erwähnt. Bei dem Versuch mit dem Chlornatriumsol wird mitgeteilt, dak bei der Kontrollprobe "eine ganz kleine Menge" präzipitiert war; ob dieser Versuch stattfand unter vollkommen denselben Umständen, als derVersuch ohne Radium, wird nicht angegeben.

Ein Kontrollversuch mit Natriumsilikatlösung ohne Radium wird nicht beschrieben "\%.

Wahrend man aus C. Doelter's Mitteilung, daB er ein "Fläschchen" mit Radiumchlorid gebrauchte ${ }^{12}$ ), folgern wïrde, dabj eine Wirkung von $\alpha$-Teilchen ausgeschlossen wäre $10.1 \mathrm{~mm}$ Glas hät ja diese vollkommen zurück), sagt er noch, als er das negative Resultat bej Aluminiumhydroxydsol mitteilt: "L'm nur die $\beta$ - bezw. die $\gamma$-Strahlen des Radiums wirken zu lassen, wurde ein Röhrchen mit demselben Inhalt wie das eben erwähnte in cine dicke Stanniolschicht eingehüllt, um die a-Strahlen gänzlich abzuhalten, und gleichzeitig mit dem vorigen dem Kadium ausgesetzi. Auch hier war das Resultat ein negatives."

Allgemeine Schlüsse werden von C. Doelter aus seinen Versuchen nicht gezogen.

2. Für unsere eigenen Versuche, betreffend den EinfluB von Radiumstrahlen auf Sole, konnten wir nur $5 \mathrm{mg}$, reines" Radiumbromid aus der Fabrik ron Dr. R. Sthamer in Hamburg anwenden, und dabei noch drei Präparate, die Herr Dr. A. Brester in Delft uns mit großer Liebenswürdigkeit zur Verfügung gestellt hatte. $\mathrm{Zu}$ samnien hatten wir aber nicht viel mehr als $15 \mathrm{mg}$ wirklich reines $\mathrm{RaBr}_{2}$, also viel weniger als die anderen Untersurher.

Dieselben in dünnem Glas eingeschmolzenen Präparate hatten jedoch früher eine deutliche Wirkung gezeigt auf Chlorknallgas ${ }^{13}$ ) und Jodo-

11) Man findet drei Angaben tibet das Koagulieren: 1, c. 22, Das kolloide Natriumsilikat wird durch Radium in der Farbe nicht verändert, dagegen war die Kieselsaure vollig gelatiniert*. 1. c. 28 , Natriumsilikat rein, in Losung als Sol, wird durch Radium zum Ge'atinieren gebracht. Das Gel wird etwas bräunlich*. 1. c. 95. "Natriumsilikatlösung wird durch Radium zers"zt, es fallt das Kieselsäurekolloid vollkommen mit veißer Farbe".

12) 1. c. 11.

19) W. P. Jorissen und W. E. Ringer, Chem. Weekbl. 2, 41 (1905); 3, 457 (1906); Ber. d. Deutsch. chem. Ges. 38, 899 (1905); 39, 2093 (1905); Arcr neerl. (2) 12, 157 (1907) form, in Chloroform gelöst ${ }^{14}$ ), und es war also möglich, daß unter günstigen Umstünden auch eine Wirkung auf ein positives Sol zu beobachten wäre.

3. Von einem Eisenoxydsol, bereitet durch Dialyse einer mit Eisenoxyd digerierten Eisenchloridlösung (Gehalt $5,9 \mathrm{~g}$ pro Liter), wurde ein Teil der Einwirkung der obengenannten in dünnes Glas geschlossenen Radiumpräparate ausgesetzt. Ein anderer Teil wurde bei derselben Temperatur geschützt vor diesem Einflub bewahrt. Beide geschlossenen Gefäße mit Sol wurden, umgeben von mehreren Glaswänden und ïberdies noch getrennt mittels einer dicken Bleiwand, bei nur wenig variierender Temperatur in einem geschlossenen Schrank aufbewahrt. Nach zwölf Tagen wurden sechs Tropfen beider Sole in je zwei Reagenzröhrchen mit flachem Boden. $20 \mathrm{ccm}$ destilliertes Wasser enthaltend gebracht. Bei Beobachtung von beiden Lösungen, während das Licht durch den Boden eintrat, konnte kein Unterschied in der Farbe beobachtet werden. Dasselbe Resultat wurde bekommen bei einem während vier Monaten dem Radium ausgesetzten Eisenoxydsol.

4. Bei einem anderen, jedoch nach gleicher Weise bereiteten Eisenoxydsol $(2,17 \mathrm{~g}$ pro Liter) wurde die Verdünnung einer Natriumnitratiösung bestimmt, die eben Koagulation hervorbrachte. Ein gewisses Volum der Natriumnitratlösung wurde jedesmal mit destilliertem Wasser verdünnt bis auf $100 \mathrm{ccm}$; dann wurden sechs Tropfen des Sols zugefügt. Ebenfalls wurden sechs Tropfen zu $100 \mathrm{ccm}$ desselben destillierten Wassers gefügt. Von beiden Flüssigkeiten wurden nach fünf Minuten die Farben in Reagenzröhrchen mit flachem Boden beobachtet idas. Licht trat ein durch den Boden). Gefunden wurde, daß die Grenze lag bei 2,82 Millimol Natriumnitrat pro ein Liter.

Es wurde nun eine Natriumnitratlösung genommen unter dieser Grenze (nämlich 1.88 Millimol pro ein Liter). $\mathrm{Zu}$ dieser wurden wieder sechs Tropfen pro $100 \mathrm{ccm}$ zugefügt. Die eine Hallte dieser Lösung wurde der Wirkung der Radiumröhrchen ausgesetzt, die andere Hälfte wurde unter möglichst gleichen Umständen außerhalb dieses Einflusses bewahrt. So wurde auch eine gleiche Zahl derselben Glusröhrchen. jedoch ohne Radium. in diese 2 weite Halfte der Lösung gebracht.

14) W. P. Jorissen und W. E. Ringer, Chem. Weekbl. 2, 799 (1005): Arch. néerl. (2) 12, 173 (1907) 
Nach einigen Versuchen, bei welchen in Abwesenheit des Beohachters beide Lösungen koagulierten. wurde gefunden, daß nach 20 Stunden die Lösung mit den Radiumröhrchen lioaguliert war, wahrend die andere klar geblieben war.

Bei einem in derselben Weise behandelten Silbersol (bereitet nach W. Muthmann, dialysiert während 13 Tagen $0,385 \mathrm{~g}$ pro ein Literi konnte niemals ein Unterschied in der Koagulationsgeschwindigkeit beobachtet werden.

5 . Während durch dieseVersuche das Resultat von Victor Henri und André Mayer bestätigt wurde, entstand die Frage, ob vorherige Behandlung mit Radiumstrahlen das Eisenoxydsol empfindlicher macht für Elektrolyte.

Für diesen Zweck wurde eine kleine Menge des unter 3 genannten Eisenoxydsols während beinahe vier Monaten dem Radiumeinflusse ausgesetzt, während eine zweite Menge aukerhalb dieses Einflusses, aber übrigens unter denselben Umständen (siehe unter 3) aufbewahrt wurde.

Auch hier befanden sich in der zweiten Menge die unter 4 genannten Röhrchen ohne Radium.

Die Untersuchung der beiden Sole geschah in folgender Weise :

Zwei Wägeröhrchen von möglichst gleichen Dimensionen wurden gewählt. In das eine wurden $3 \mathrm{ccm}$ destilliertes Wasser. in das andere $3 \mathrm{ccm}$ Salzlösung gebracht. In beide wurde $0.02 \mathrm{ccm}$ Sol gebracht. Nach dem Mischen wurde eine Minute gewartet und dann wurden die beiden Röhrchen gegen das Licht beobachtet. War die Salzlösung trübe geworden, so wurde eine mehr verdünnte genommen und, also weitergehend, wurde endlich eine Lösung gefunden, welche eben Koagulation verursachte und eine andere, welche unter denselben Umständen gerade keine Koagulation gab. Das Mittel aus beiden Konzentrationen wurde als Grenze betrachtet. Diese Konzentration ist ein Mab für die Enipfindlichkeit des Sols gegen Elektrolyte.

Gefunden wurde, daß die Grenze für Kaliumsulfat bei dem bestrahlten Sol 0,095 Millimol pro Liter war, und bei dem nichthestrahiten Sol 0,13 Millimol pro Liter. Der Unterschied ist ziemlich groß.

6. Eine neue Menge desselben Sols wúrde jetzt über einen Monat dem Radium ausgesetzt, in derselben Weise wie oben beschrieben. Die Resultate findet man in folgender Tabelle.

\begin{tabular}{|c|c|c|}
\hline \multirow[b]{2}{*}{ S:llt } & \multicolumn{2}{|c|}{ Grenze } \\
\hline & $\begin{array}{c}\text { für das } \\
\text { bestrahlte Sol in } \\
\text { Millimolen pro Liter }\end{array}$ & $\begin{array}{l}\text { für das } \\
\text { inichtbestrahlte Sol in } \\
\text { Millimnolen pro Liter }\end{array}$ \\
\hline $\mathrm{K}_{2} \mathrm{C}_{z} \mathrm{O}_{4}$ & 0,027 & 0.035 \\
\hline $\mathrm{K}, \mathrm{CO}_{3}$ & 0,24 & $n .26$ \\
\hline $\mathrm{KCl}$ & 44,0 & 44.5 \\
\hline $\mathrm{NaCl}$ & 46,4 & 46,4 \\
\hline $\mathrm{Na}_{2} \mathrm{CO}_{3}$ & 0,22 & 0,16 \\
\hline Na-Zitrat & 0,0095 & 0,0090 \\
\hline $\mathrm{CuSO}_{4}$ & 0.13 & 0.17 \\
\hline $\mathrm{MgSO}_{4}$ & 0,13 & 0,16 \\
\hline $\mathrm{MnSO}_{4}$ & 0,18 & 0.18 \\
\hline
\end{tabular}

Im allgemeinen ist also das bestrahlte $\mathrm{Sol}$ empiindlicher als das nichtbestrahlte. In Falle von $\mathrm{Na}$-Zitrat ist die Grenz-Konzentration so klein, daß diesen Zahlen kein großer Wert beizulegen ist. Das Resultat mit Natriumkarbonat können wir nicht erklären. Ob wir jedoch mit der uns zur Verfügung stehenden sehr kleinen Menge Radium bessere Resultate erwarten können, es sei denn mit sehr langer $\mathrm{Be}$ strahlung, muß bezweifelt werden.

Leiden und Helder (Holland), August 1910.

\section{Ultramikroskopische Beobachtungen.}

Von J. Amann, Lausanne.

(Elngegangen am 30. Nov. 1910)

\section{Kryptokinese und kryptokinetische Bewegungen.}

Diese neuen Bezeichnungen möchte ich für gewisse eigentümliche Bewegungen der ultramikroskopischen Teilchen vorschlagen, welche so schwach sind, dafs sie in der Regel nicht unmittelbar wahrgenommen werden können, sondern nur durch besondere Kunstgriffe sichtbar werden.
Bekanntlich erscheinen unter dem Ultramikroskop, bei genauer Einstellung die stark erleuchteten Teilchen in der Form eines kleinen Scheibchens, dessen Durchmesser unter anderem von der Oeffnung des optischen Systems abhängt. Mit den zur Beobachtung gebräuchlichen Trockenobjektiven von $4-6 \mathrm{~mm}$ äquivalenter Brennweite und etwa $0,6-0,7$ numerischer Apertur, besteht dieses Diffraktions- 